\title{
Verdicts of Inguinal Hernia Surgery: Report of Rare Case
}

Dr. Lalita Bansal ${ }^{1 *}$, Dr. Ashish Dhiman
${ }^{\mathrm{T}}$ Medical officer (OBG) R.H. Kullu, Himach
${ }^{2}$ Medical Officer (General Surgery), R.H. K
Article History
Received: 03.12 .2021
Accepted: 21.01 .2022
Published: 24.01 .2022
Journal homepage:
https://www.easpublisher.com

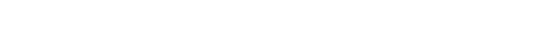
the appropriate treatment.

Keywords: Amyand's hernia, epiploicappendagitis, ovarian and tubal hernia,
}

\begin{abstract}
Background and aim: To present our experience with unexpected findings during hernia surgery, either unusual hernia contents or pathologic individuals, like neoplastic masses, masked as a hernia. Patients and methods: We studied retrospectively 550 patients with inguinal hernia who were admitted to our surgery department over a 6-year period. In addition, our study included patients complaining of inguinal protrusion, even without a definitive diagnosis of inguinal hernia upon admission. Results: Five patients presented with unusual hernial contents. Three of them had a vermiform appendix in their sac. Acute appendicitis (Amyand'shernia) was found in only one case. One patient had epiploic appendagitis related with a groin hernia. Moreover, an adult woman was diagnosed with ovarian and tubal inguinal hernia. Finally, we report a case of a massive extra testicular intra scrotal lipoma, initially misdiagnosed as a scrotal hernia. Conclusion: A hernia surgeon may encounter unexpected intraoperative findings. It is important to be prepared to detect them and apply inguinal mass \& intrascrotallipoma.
\end{abstract}

Copyright (C) 2022 The Author(s): This is an open-access article distributed under the terms of the Creative Commons Attribution 4.0 International License (CC BY-NC 4.0) which permits unrestricted use, distribution, and reproduction in any medium for non-commercial use provided the original author and source are credited.

\section{INTRODUCTION}

One of the most common operations in surgical practice is inguinal hernia repair. Despite their high prevalence, hernias can pose a surgical problem, even for the most experienced surgeon1. One of these instances is the unexpected hernial content. We describe our experience with ovary, fallopian tube, epiploicappendagitis, vermiform appendix, and acute appendicitis (Amyand's hernia) as atypical hernial contents. Furthermore, numerous clinical conditions can masquerade as inguinal hernia. It's critical to be ready to spot them and make the necessary surgical judgments. A retrospective review of the case histories of 550 inguinal hernia patients who were admitted to our department between 2015 and 2021 was undertaken. Information was obtained from their medical records and the documented operative findings. In addition, our study included patients complaining of inguinal protrusion, even without a definitive diagnosis of inguinal hernia upon admission, in an effort to present all the unexpected findings, true hernias or not, that a hernia surgeon may confront during her/his practice.

\section{Case Description}

\section{Vermiform appendix in inguinal hernia sac}

Vermiform appendix as a hernial sac content was identified in 3patients $(0.38 \%)$.One of them had

*Corresponding Author: Dr. Lalita Bansal

Medical officer (OBG) R.H. Kullu, Himachal Pradesh India acute appendicitis (Amyand'shernia) and presented as in cerebrated right groin hernia. The twopatientswithnormalappendixhadameshherniarepairw ithoutappendicectomy.InthecaseofAmyand's ernia, the patient underwent simultaneous appendicectomy and Bassini's suture hernia repair. Notably, since appendix seemed in accessible through then neck of the sac, the hernia incision was extended superiorly. No postoperative infection recurrence was reported.

\section{Epiploicappendagitis in inguinal hernia sac}

Epiploicappendagitis due to chronic torsion and inflammation secondary to an inguinal hernia occurred in one patient $(0.12 \%)$ who was admitted with a 4-month history of a left groin lump. It caused only irritation symptoms and mass formation, without cough impulse. Intra-operatively, a thick hernia sac was found and opened. An inflamed, swollen epiploic appendage of the sigmoid colon, was identified (Figure1).The sigmoid colon had normal appearance and was not found in the hernia sac. The epiploic appendage was resected and mesh-plug repair followed.

\section{Ovary and fallopian tube in inguinal hernia sac}

A 39-year-old woman was diagnosed having an ovarian and tubal inguinal hernia. It presented as irreducible indirect groin hernia. Surgical exploration revealed an edema to us but still viable left fallopian 
tube and a normal ovary in the hernia sac (Figure2).The tube and the ovary were replaced into the peritoneal cavity and mesh-plug repair was performed.

\section{A massive extra testicular intra scrotallipomamas querading as an inguinal hernia}

Another peculiar case was that of a massive primary lipoma of the scrotum, initially misdiagnosed as an inguinoscrotal hernia. Patient's chief complaint was swelling which was already present for the last eight years. Believing that he had a hernia, he did not seek any medical attention until its increase to alargesize mass. At clinical examination, an elastic, irreducible soft mass was palpated, without tenderness or Trans-illumination. No bowel sounds were detected within the scrotum. Ultrasound and CT imaging demonstrated a solid mass, most probably alipoma. The lesion was resected (Figure3). It was adherent to the scrotal wall but easily separable from it. Testis, epididymis and spermatic cord were intact. The removed specimen measured 20X16X6 cm and was enveloped into a membrane (Figure4). The histopathology revealed a lipoma.

\section{DISCUSSION}

Although rare, hernia sac may contain vermiform appendix and, exceptionally, acute appendicitis. Dr. Garengeot first reported this finding in a femoral hernia sac [2], in 1731. Subsequently, a voluminous literature evolved in which anatomists and surgeons recorded the availability of the appendix within the hernia sac. The first recorded appendicectomy was performed by Claudius Amy and in1736 through a right inguinal hernia sac [3-5]. The patient was an 11-year-old boy with an appendiceal abscess in the scrotum. The operation was unsuccessful because of recurrent sepsis following the removal of the appendix and its contained foreign bodies.

Acute appendicitis with in an inguinal hernia accounts for $0.1 \%$ of all cases [6]. Inflammation of the appendix is attributed to the external compression of the appendix at the neck of the hernia. The inflammatory status of the vermiform appendix determines the surgical approach and the type of hernia repair. In the case of appendicitis due to the infective and inflammatory process, hernia repair should be performed without synthetic meshes and plugs within the defect. In the case of a normal appendix incidentally found within the sac, the addition of prophylactic appendicectomy to the hernia repair is not favored. Appendicectomy adds the possibility of infection to an otherwise clean procedure. Superficial wound infection adds morbidity; deep infection may contribute to recurrence. In addition, surgical efforts to achieve visualization of the entire appendix, by enlarging the hernial defect or distending the neck of the sac, increase the possibility of recurrence, by weakening of the anatomic but tress around a rupture [5].
The epiploic appendages are 2-5 cm long, fat containing out pouchings of peritoneum of the large bowel, arranged to the tenialibera medially and to the teniaomental is laterally. Epiploic appendages may be involved in a number of disease processes such as epiploic appendage it is due to torsion or venous occlusion or acute and chronic inflammation secondary to diverticulitis. When found in the inguinal hernia sac, the epiploic appendages may appear normal, hypertrophied, inflamed with diverticulitis, or strangulated with necrosis. Epiploic appendagitis as an inguinal hernia content is very rare. Only two cases have been reported in MEDLINE, one from Turkey [7] and the other one from Russia [8]. Earlier cases on relation of epiploic appendages and hernia have been reported from USA as early as the 1920s [7, 9]. Although epiploic appendagitis is a self-limiting condition, many times operation is unavoidable in order to rule out a possible in carcerated hernia. Imaging tools like ultrasonography may not be helpful in discriminating an inflamed epiploic appendage from the partly superposed colon. The mass could be misdiagnosed as a bowel loop and the pathological entity as an in carcerated bowel loop. In addition, an apparent inguinal mass must be excised, because many pathologic conditions (some of them malignant) can masquerade as inguinal hernia. So, the treatment consists of excision of the epiploic appendage with an attention to avoid opening of aconcominant diverticulum. Tension-free repair of the hernia follows. Sac should only be attempted if no ovarian or tubal abnormality is present and the blood supply is not impaired.

Intrascrotal lipoma is not high in the differential diagnosis of an in guino scrotal mass. Benign lipomatous tumors are not common in the scrotum. Although not found in a hernia sac, they can mimic irreducible groin hernias. Scrotal lipomas are classified into 3 types depending on their site of origin and spread those arising from the fatty tissue within the spermatic cord and spreading to the scrotum, those originating and developing within the spermatic cord and those originating and developing with in the scrotal walls (primary lipoma of the scrotum). The second type is the most common and account for about half of all cord tumors, while the third type, the primary lipoma of the scrotum, is rare $[14,15]$. In our case, the lipoma seemed to originate from the subcutaneous tissues posterior to the spermatic cord and spread into the scrotum. Despite to similar to our already reported cases, giant tumors, as the one described here, are not common $[15,16]$. In conclusion, a hernia surgeon may encounter unexpected intraoperative findings. It is important to be prepared to detect them and apply the appropriate treatment. 


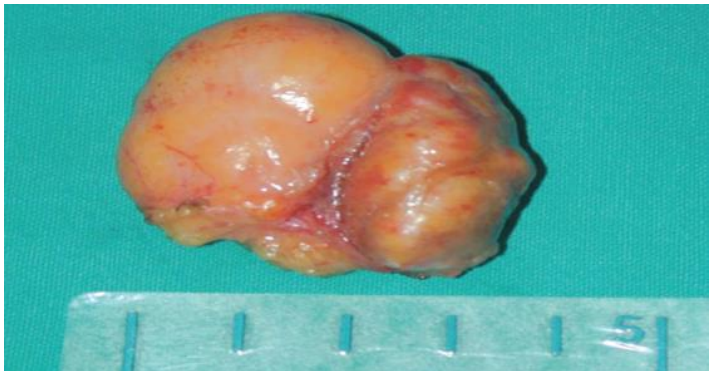

Fig-1: An inflamed appendage epiploic a of the sigmoid colon, as a hernia sac content

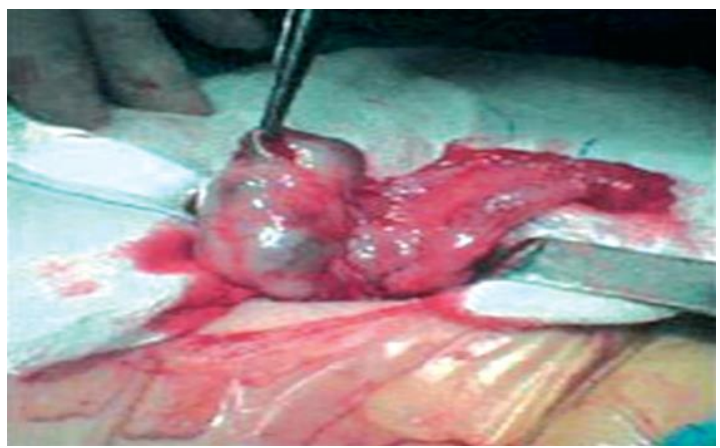

Fig-2: Ovarian and tubal inguinal hernia in an adult patient

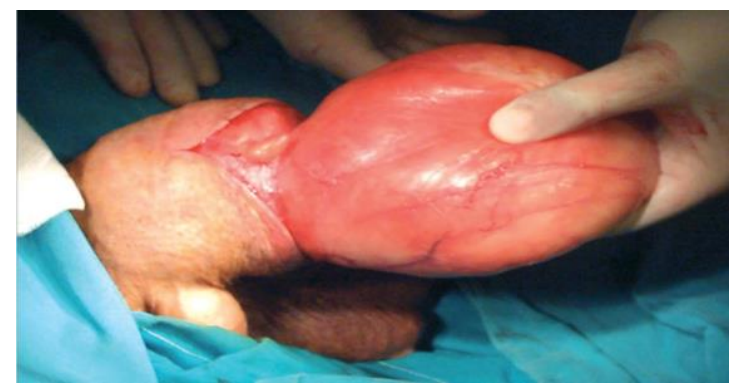

Fig-3: Massive intrascrotal extratesticular lipoma

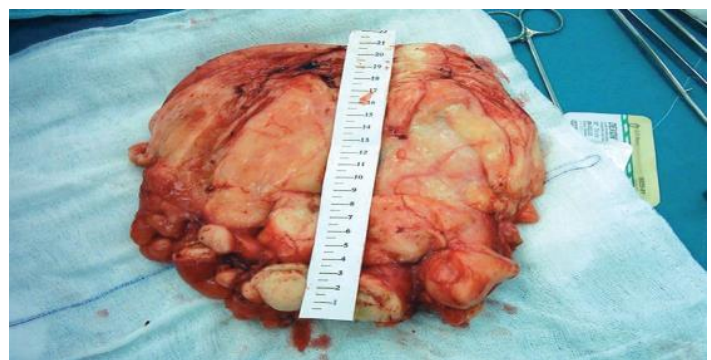

Fig-4: Massive intra scrotal extra testicular lipoma. The removed specimen

\section{REFERENCES}

1. IM, R. (1993). Robbins AW. Demographic, classificatory, and socioeconomic aspects of hernia repair in the United States. Surg Clin North Am, 73, 413-426.

2. Akopian, G., \& Alexander, M. (2005). De
Garengeot hernia: appendicitis within a femoral hernia. The American Surgeon, 71(6), 526-527.

3. Amyand, C. Of an inguinal rupture, with a pin in the appendixcaeci, incrustedwith stone; and some observations on wounds in the guts. Philosophical Transactions of the Royal Society of London. 1736; 39: 329-336.

4. Hutchinson, R. (1993). Amyand'shernia. JRSocMed, 86; 104-105.

5. Solecki, R., Matyja, A., \& Milanowski, W. (2003). Amyand's hernia: a report of two cases. Hernia, 7(1), 50-51.

6. Gurer, A., Ozdogan, M., Ozlem, N., Yildirim, A., Kulacoglu, H., \& Aydin, R. (2006). Uncommon content in groin hernia sac. Hernia, 10(2), 152-155.

7. Kulacoglu, H., Tumer, H., Aktimur, R., \& Kusdemir, A. (2005). Epiploic appendicitis in inguinal hernia sac presenting an inguinal mass. Hernia, 9(3), 288-290.

8. Abdulzhavadov, I. M. (1989). Volvulus of the epiploic appendices of the sigmoid in strangulated inguinal hernia. Klinicheskaia meditsina, 67(1), 126-127.

9. Kingenstein, P. (1924). Some phases of the pathology of the appendices epiploicae. Surg Gynoecia Obstet, 38, 376-382.

10. Heineck, A. P. (1912). Hernias of the ovary, of the fallopian tube, and of the ovary and the fallopian tube: An analytical review of all undoubted cases of this nature reported in the French, German, and English medical literature from 1890 to 1910 , inclusive, with a report of unpublished cases. Surg Gynecol Obstet, 15, 63-71.

11. MAYER, V., \& TEMPLETON, F. G. (1941). Inguinal ectopia of the ovary and fallopian tube: review of the literature and report of the case of an infant. Archives of Surgery, 43(3), 397-408.

12. Bradshaw, K. D., \& Carr, B. R. (1986). Ovarian and tubal inguinal hernia. Obstetrics and gynecology, 68(3 Suppl), 50S-52S.

13. Amarin, Z. O., \& Hart, D. M. (1988). Inguinal ovary and fallopian tube-an unusual hernia. International Journal of Gynecology \& Obstetrics, 27(1), 141-143.

14. Leyson, J. F. J., Doroshow, L. W., \& Robbins, M. A. (1976). Extratesticular lipoma: report of 2 cases and a new classification. The Journal of urology, 116(3), 324-326.

15. Zeng, L., Xia, T., Kong, X., \& Gu, F. (1999). Massive lipoma of the scrotum. Chinese medical journal, 112(1), 84-85.

16. Greeley Jr, D. J., Sullivan, J. G., \& Wolfe, G. R. (1995). Massive primary lipoma of the scrotum. The American Surgeon, 61(11), 954-955.

Cite This Article: Lalita Bansal \& Ashish Dhiman (2022). Verdicts of Inguinal Hernia Surgery: Report of Rare Case. East African Scholars J Med Surg, 4(1), 24-26. 